\title{
Gambling Harms: A Dominance Analysis of Cognitions, Motivation and Impulsivity
}

\author{
Alysha Cooper and Harvey HC Marmurek* \\ University of Guelph Guelph, Ontario, Canada N1G 2W1
}

\author{
${ }^{*}$ Corresponding author \\ Harvey HC Marmurek, Department of Psychology, University of Guelph, \\ Guelph, Ontario, Canada N1G 2W1
}

Submitted: 12 Jul 2020; Accepted: 16 Jul 2020; Published: 27 Jul 2020

\begin{abstract}
Explanatory models of substance and behavioral addictions typically emphasize the contributions of three predictor domains: distorted cognitions related to control; motivations related to rewards and stress-reduction; and, failure to regulate emotions. In the present study, 271 (161 females) patrons at a racetrack-slots facility completed a survey comprising standardized measures of gambling-related cognitions, motivations for gambling, trait impulsivity, and problem gambling severity. The purpose was to explore dominance analysis as a statistical procedure to identify the relative importance of the three domains as predictors of the experience of gambling harms. The first step of the analysis isolated the dominant facet within each of the three multi-dimensional domains. The final step computed relative dominance among those facets. The results indicated that the most dominant predictor was the cognition of an inability to stop gambling. Motivation to avoid life stressors was the second most dominant predictor followed by the tendency to act rashly in the presence of negative emotion (negative urgency). The relative dominance of the predictors of gambling harm may provide a framework for scaffolding interventions directed at mitigating gambling harms.
\end{abstract}

Keywords: Gambling Harms; Cognitions; Motivation; Impulsivity; Dominance Analysis

\section{Introduction}

Gambling is a popular recreational activity. In a review of gambling prevalence across 31 countries, Calado and Griffiths reported a range from 25.5\% (Czech Republic) to 86\% (New Zealand) [1]. The incidence of problem gambling, however, was relatively low $(0.12 \%$ to $5.8 \%)$. The wide variation in estimates of gambling prevalence and problem gambling reflects differences in methodologies, measurement instruments, and cultural trends. Canale et al. noted that although problem gamblers report more harms than non-problem gamblers, at the population level there are more harms from non-problem gamblers as they are much more frequent in the population. This leads to a "prevention paradox" if the goal of treatment and policy is directed at the extreme cases [2].

Research focused on harm reduction has sought to identify predictors of harmful gambling. Socio-demographic predictors of problem gambling include age, education, gender, ethnicity, marital status, and income (Calado \& Griffiths) [1]. Beyond sociodemographic factors, researchers have explored the relation between problem gambling and specific gambling activities. Delfabbro et al. examined the association between problem gambling and three types of activities: electronic gaming machines (EGMs); racing; and, casino table games [3]. Their data pooled the results from approximately 100,000 respondents across 12 prevalence studies conducted in Australia between 2011 and 2020. The rate of problem gambling, measured by the Problem Gambling Severity Index (PGSI) (Ferris \& Wynne) [4], was 0.65\%. Overall, the data indicated a strong association between problem gambling and gambling on EGMs. For example, whereas $21.2 \%$ of the full sample reported betting on EGMs, $81.7 \%$ of problem gamblers reported betting on EGMs [3]. Delfabbro et al. noted that problem gamblers are more likely to participate in a wider range of gambling activities than are non-problem gamblers, and they attributed that pattern to the characteristics of the gambler rather than the individual activity [3]. Similarly, Nelson et al. suggested that gambling patterns may be influenced by the interaction of game characteristics and personal characteristics [5].

Many personality and psychosocial characteristics have been associated with disordered gambling. In a comprehensive analysis of 23 predictors of problem gambling severity, Chiu and Storm found that trait impulsivity was the strongest predictor [6]. The role of impulsivity on problem gambling has been supported in many studies (MacLaren et al., 2011; Michalczuk et al., 2011; Odlaug et al., 2013; Passanisi \& Pace) [7-10]. Gambling is also sustained by distorted gambling cognitions (Devos et al., Goodie \& Fortune, Myrseth et al.,) [11-13]. Devos et al. identified five clusters of gambler types differentiated by facets of impulsivity and cognitive distortion [11]. For example, the two clusters that were most likely to seek treatment were: impulsive emotional 
gamblers characterized by high urgency and inability to control gambling; and, impulsive gamblers with gambling-related cognitions who had high levels of sensation seeking and illusion of control. The influence of impulsivity and cognitions on gambling severity is modulated by different motivations for gambling. Hearn et al. distinguished three types of gamblers:social gamblers who are motivated by socialization; affect-regulation gamblers who seek to control negative affective states; and, antisocial gamblers who are motivated by excitement [14].

A practical implication of recognizing the heterogeneity of gamblers is to tailor interventions according to the constellation of facets of impulsivity, cognitions and motivation presented by the gambler (Devos et al.) [11]. The current study addressed the relative importance of those factors to the harms resulting from gambling. We measured gambling harms with a subset of items from the PGSI (Ferris \& Wynne) [4]. The PGSI, considered the gold standard of problem gambling screening instruments (Caler et al., Miller et al.,) comprises items that may be categorized as either behaviours or adverse consequences [15, 16]. Harm was indexed by the scores on the adverse consequence items. Dominance analyses (Azen \& Budescu), based on the regression of harm against multi-dimensional measures of impulsivity, gambling cognitions and gambling motivations measured the relative importance of those factors as predictors of gambling harms [17].

Dominance analysis is designed to address the problem that common measures of "importance" (e.g., $r, \beta$ ) may not yield consistent orderings of the importance of a set of predictors. In dominance analysis, one predictor is considered more important than another "if it contributes more to the prediction of the criterion than does its competitor at a given level of analysis" (Azen \& Budescu, p. 133) [17]. The level of analysis refers to the number of predictors in a model (e.g., $r$ reflects a null model in which all other predictors are excluded, and $\beta$ reflects a full model in which all predictors are present). For any set of predictors, there are $2^{\mathrm{p}}-1$ subset regression models of which $2^{\mathrm{p}-2}$ can be used to determine dominance for [p(p$1)] / 2$ pairs of predictors. Thus, for five predictors there are eight subset regression models which can be used to determine dominance and 10 distinct pairings of predictors. Two predictors, $X_{1}$ and $X_{2}$, can be compared for dominance on all model subsets made up of the other predictors. For comparison, the additional contribution of each of the two predictors to the fit of the model subsets which exclude both of the predictors is found. The additional contribution to the model fit is measured by the increase in the proportion of variance in the criterion accounted for by the model if the predictor of interest were to be added to the model.

If one predictor $\left(\mathrm{X}_{1}\right)$ increases the proportion of variance accounted for in the criterion more so than another predictor $\left(\mathrm{X}_{2}\right)$ for all of the models for which they can be compared, then $\mathrm{X}_{1}$ is said to completely dominate $\mathrm{X}_{2}$; that is, $\mathrm{X}_{1}$ is considered to be more important than $X_{2}$. The dominance relationship between $X_{1}$ and $X_{2}$ may be represented numerically with one of three values: 1 if $X_{1}$ dominates $\mathrm{X}_{2} ; 0$ if $\mathrm{X}_{2}$ dominates $\mathrm{X} 1$; and, 0.5 if the dominance relationship is indeterminate. A bootstrapping procedure underlies inferences about the reproducibility of the dominance result. The current study utilized dominance analysis to yield knowledge about the relative importance of predictors of gambling harms and may provide a useful guide in structuring interventions.

\section{Method}

\section{Participants}

This research project received ethics approval from the Research Ethics Board at the authors' university. The participants were 271 (161 females) patrons at a racetrack-slots facility in Ontario, Canada. Ages ranged from 21 to $71(M=46.61, S D=13.01)$. A recruitment poster was placed adjacent to the entrance to the gambling area. The poster stated that a research team was on the premises to study "the characteristics of gamblers." The poster indicated that participants would be paid $\$ 30$ for their participation. Patrons who wished to participate were directed by a member of the research team to a room outside the gambling area where they completed a survey containing a series of standardized scales measuring problem gambling severity, trait impulsivity, gambling related cognitions, and gambling motivation. The survey was presented electronically or in paper format according to the preference of the participant.

\section{Measurement instruments \\ Problem gambling}

The Problem Gambling Severity Index (PGSI) is a nine-item subset of the Canadian Problem Gambling Inventory (Ferris \& Wynne) [4]. Respondents are asked to think about the past year and to indicate the frequency for each item using a 4-point scale: 0 = never; 1 = sometimes; $2=$ most of the time; 3 = almost always. The nine items are: How often have you bet more than you could really afford to lose? How often have you needed to gamble with larger amounts of money to get the same feeling of excitement? How often have you gone back another day to try to win back the money you lost? How often have you borrowed money or sold anything to get money to gamble? How often have you felt that you might have a problem with gambling? How often have people criticized your betting or told you that you had a gambling problem, regardless of whether or not you thought it was true? How often have you felt guilty about the way you gamble or what happens when you gamble? How often has your gambling caused you any health problems, including stress or anxiety? How often has your gambling caused any financial problems for you or your household?

Whereas the first four PGSI items refer to problem gambling behaviours, the final five items refer to adverse consequences (Wynne) [18]. The original scoring of the PGSI classified four gambling subtypes on the basis of the sum of the responses across the nine items:0 = non-problem gambler; 1-2 = low-risk gambler; 3-7 = moderate-risk gambler; 8 or more $=$ problem gambler. Nonproblem and low-risk gamblers were described as not having experienced any adverse consequences from gambling. Revised cutoffs (Currie et al.,) of 1-4 and 5-7 provided better distinctiveness between the middle categories [19]. For the current sample, 
Cronbach's alpha for the 9-item PGSI $(M=2.34 ; S D=3.09)$ was 0.82 Cronbach's alpha for the 5-item Harm scale comprising the adverse consequences items $(M=1.07 ; S D=1.73)$ was 0.74 .

\section{Impulsivity}

Cyders et al. identified five facets of impulsivity as follows: lack of planning (e.g., disagreement on "I like to stop and think things over before I do them"); lack of perseverance (e.g., "There are so many little jobs that need to be done that I sometimes just ignore them all"); sensation seeking (e.g., "I sometimes like doing things that are a bit frightening"); negative urgency (e.g., "I often make matters worse because I act without thinking when I am upset"); and, positive urgency (e.g., "When overjoyed, I feel like I can't stop myself from going overboard") [20]. The impulsivity scale (Cyders et al.,) contains 59 items for which respondents indicate their level of agreement using a 4-point scale from $1=$ agree strongly to $4=$ disagree strongly [20]. The Cronbach's alpha indices of internal consistency for the separate factors in the current study were: lack of planning, .86; lack of perseverance, .85; sensation seeking, .91; positive urgency, .96; and, negative urgency, .90 .

\section{Gambling Motivation}

The Gambling Motivation Scale (Lee et al.,) asks respondents to indicate their level of agreement with 27 statements using a 5-point scale where $1=$ strongly disagree, $3=$ neutral, and $5=$ strongly agree [21]. The scale identifies five separate factors: excitement (e.g., "have fun in risk-taking"); monetary (e.g., "make money easily"); avoidance (e.g., 'feel lonely/escape from loneliness"); socialization (e.g., "socialize with others"); and, amusement (e.g., "enjoy leisure time and activity"). The Cronbach's alpha for the five separate factors in the present study were: amusement, 0.72; avoidance, 0.87 ; excitement, 0.91; monetary, 0.75; and, socialization, 0.81 .

\section{Gambling Cognitions}

Raylu and Oei developed The Gambling Related Cognition Scale (GRCS) which comprises 23 items [22]. Participants indicate their level of agreement with each statement on a 7-point scale $(1=$ strongly disagree; $7=$ strongly agree). The GRCS identifies five cognitive factors related to gambling: expectancies (e.g., "gambling makes the future brighter"); illusion of control (e.g., "specific numbers and colors can help increase my chances of winning"); predictive control (e.g., "losses when gambling is bound to be followed by a series of wins"); inability to stop (e.g., "I can't function without gambling"); and, interpretive bias (e.g., "relating my losses to probability makes me continue gambling"). In the present study, Cronbach's alpha values for the subscales were as follows: expectancies, 0.82 ; illusion of control, 0.77; predictive control, 0.77 ; inability to stop, 0.87 ; interpretive bias, 0.85 .

\section{Results}

PGSI Analyses

The original scoring criteria setting 3 as the cut-off between low-risk and moderate-risk gamblers indicated the following distribution of participants across gambling severity categories: non-problem gambler, 92 (34\%); low-risk gambler, 89 (33\%); moderate-risk gambler, 72 (27\%); and, problem gambler, 18 (7\%). If the cut-off value for moderate-risk gambler were 5 rather than 3 , then the number of low-risk gamblers is increased to $135(50 \%)$ and the number of moderate-risk gamblers is reduced to $26(10 \%)$. PGSI scores were significantly skewed, Shapiro-Wilk $=0.74, p<.001$. Non-parametric tests showed that difference between male $(M=$ $2.54, S D=3.22)$ and female $(M=2.19, S D=2.99)$ scores was not statistically significant, $U=8.11, p=.23$, and the correlation between age and PGSI also was not statistically significant, $r h o=.09, p=.11$. Similar patterns occurred for the Harm scores (the final five items on the PGSI scale) where skewness was statistically significant, ShapiroWilk $=0.65, p<.001$.Non-parametric tests showed that the difference between Harm scores for males $(M=1.13, S D=1.89)$ and females $(M=1.02, S D=1.62)$ was not statistically significant, $U=8403, p=$ .44 , and the correlation between age and Harm was not statistically significant, $r h o=.08, p=.18$.

\section{Overview of Dominance Analysis}

As noted above, the distribution of Harm scores based on five items on the PGSI was significantly skewed. Dominance analysis assumes a normal distribution such that a violation of the assumption could lead to incorrect conclusions and interpretations regarding the relationship between variables (Delaney, 2010; Vargha, Bergman, \& Delaney) [23, 24]. Therefore, we conducted the dominance analyses for a logistic regression where the dependent variable indicated whether or not an individual experienced any harms (i.e., Harm score $=0$ vs. Harm score $>0)$. There were $128(47 \%)$ participants with a Harm score greater than 0 . For dominance analysis with logistic regression, a variation of $R^{2}$, known as $R^{2}$ (McFadden, 1974), is recommended as the measure of model fit (Azen \& Traxel) $[25,26] . R_{m}^{2}$ is bounded between 0 and 1 , with 0 indicating a poor fitting model and 1 indicating a perfect fitting model. $R_{m}^{2}$ can roughly be interpreted as the proportional reduction in error variance for the model with the predictors versus the null model (i.e., the model with no predictors) (Menard) [27].

To determine the relative importance of all 15 facets across the three factors simultaneously would require testing 8,192 regression subset models and 105 pairings of predictors. To render the presentation of results more tractable, we report the dominance analyses for the 10 pairings of the five facets separately for impulsivity, gambling motivation, and gambling cognitions. Next, we report the model fit for the seven subset regression models and the full model of the three dominant facets across those factors. Of these model subsets, dominance between the three pairings of the facets can be determined on four of the models.

\section{Dominance Analysis of Impulsivity Facets}

The summary of the dominance analysis for the facets of impulsivity with bootstrapping can be found in Table 1. The first two columns in the table present the variables compared for dominance. The following column presents the dominance scores on the original sample. The dominance score $\left(D_{i j}\right)$ is equal to 1 if the variable in the first column completely dominated the variable in the second column, is equal to 0.5 if complete dominance could not be established, or is equal to 0 if the variable in the second 
column completely dominated the variable in the first column. The fourth and fifth columns present the mean dominance score (i.e., $\bar{D}_{i j}$ )and standard error of the dominance (i.e., $S E\left(D_{i j}\right)$ ), respectively, across 1,000 bootstrap samples. The proportion of each dominance score observed across the bootstrap samples for the two variables is found in the following three columns. P $i j$ is the proportion of samples which had the variable in the first column completely dominate the variable in the second column, P $j i$ is the proportion of samples which had the variable in the second column completely dominate the variable in the first column, and $\mathrm{P}_{\text {noij }}$ is the proportion of samples which did not establish dominance between the two variables.
Table 1 shows that positive urgency, negative urgency, and sensation seeking each dominated perseverance and planning. In the original sample, dominance could not be determined among positive urgency, negative urgency, and sensation seeking. However, in repeated sampling, negative urgency dominated positive urgency $43.3 \%$ of the time whereas positive urgency dominated negative urgency $35.5 \%$ of the time, indicating that negative urgency is more dominant than positive urgency. Furthermore, in repeated sampling, negative urgency dominated sensation seeking $23.2 \%$ of the time whereas sensation seeking dominated negative urgency $15.2 \%$ of the time, suggesting that negative urgency is more dominant than sensation seeking.

\section{Table 1}

Logistic dominance analysis of impulsivity facets on gambling harm. $D_{i j}$ values in the sample and their means $\left(\bar{D}_{i j}\right)$, standard errors, probabilities of dominating one another, and reproducibility over $S=1,000$ Bootstrap Samples.

\begin{tabular}{|c|c|c|c|c|c|c|c|}
\hline I & $\mathbf{j}$ & $D_{i j}$ & $\bar{D}_{i j}$ & $S E\left(D_{i j}\right)$ & $\mathbf{P}_{\mathrm{ij}}$ & $\mathbf{P}_{\mathrm{ji}}$ & $\mathbf{P}_{\text {noij }}$ \\
\hline Positive Urgency & Negative Urgency & 0.5 & 0.461 & 0.442 & 0.355 & 0.433 & 0.212 \\
\hline Positive Urgency & Sensation Seeking & 0.5 & 0.511 & 0.323 & 0.220 & 0.197 & 0.583 \\
\hline Positive Urgency & Planning & 1 & 0.745 & 0.266 & 0.506 & 0.016 & 0.478 \\
\hline Positive Urgency & Perseverance & 1 & 0.782 & 0.250 & 0.567 & 0.002 & 0.431 \\
\hline Negative Urgency & Sensation Seeking & 0.5 & 0.540 & 0.307 & 0.232 & 0.152 & 0.616 \\
\hline Negative Urgency & Planning & 1 & 0.788 & 0.268 & 0.599 & 0.022 & 0.379 \\
\hline Negative Urgency & Perseverance & 1 & 0.823 & 0.244 & 0.651 & 0.005 & 0.344 \\
\hline Sensation Seeking & Planning & 1 & 0.812 & 0.311 & 0.701 & 0.076 & 0.223 \\
\hline Sensation Seeking & Perseverance & 1 & 0.857 & 0.272 & 0.759 & 0.046 & 0.195 \\
\hline Planning & Perseverance & 0.5 & 0.543 & 0.375 & 0.329 & 0.242 & 0.429 \\
\hline
\end{tabular}

\section{Dominance Analysis of Motivation Facets}

The summary for the dominance analysis with bootstrapping for gambling motivation facets is presented in Table 2. Motivation for avoidance and money both dominated motivation for excitement, socialization, and amusement. In the original sample, dominance could not be established between motivation for avoidance and motivation for money. Repeated sampling showed that avoidance dominated money more often $\left(\mathrm{P}_{\mathrm{ij}}=0.410\right)$ than money dominated avoidance $\left(\mathrm{P}_{\mathrm{ji}}=0.362\right)$, indicating that motivation for avoidance is more dominant than motivation for money. Motivation for amusement dominated motivation for socialization but dominance could not be determined between amusement and excitement. Finally, dominance between excitement and socialization could not be established.

Table 2

Logistic dominance analysis of gambling motivation facets on gambling harm. $D_{i j}$ values in the sample and their means $\bar{D}_{i j}$, standard errors, probabilities of dominating one another, and reproducibility over $S=1,000$ Bootstrap Samples.

\begin{tabular}{|c|c|c|c|c|c|c|c|}
\hline $\mathbf{i}$ & j & $D_{i j}$ & $\bar{D}_{i j}$ & $S E\left(D_{i j}\right)$ & $\mathbf{P}_{\mathrm{ij}}$ & $\mathbf{P}_{\mathrm{ji}}$ & $\mathbf{P}_{\text {noij }}$ \\
\hline Avoidance & Money & 0.5 & 0.524 & 0.439 & 0.410 & 0.362 & 0.228 \\
\hline Avoidance & Amusement & 1 & 0.743 & 0.343 & 0.595 & 0.110 & 0.295 \\
\hline Avoidance & Excitement & 1 & 0.828 & 0.238 & 0.655 & 0.000 & 0.345 \\
\hline Avoidance & Socialization & 1 & 0.884 & 0.211 & 0.769 & 0.000 & 0.231 \\
\hline Money & Amusement & 1 & 0.748 & 0.366 & 0.639 & 0.143 & 0.218 \\
\hline Money & Excitement & 1 & 0.852 & 0.228 & 0.704 & 0.000 & 0.296 \\
\hline Money & Socialization & 1 & 0.875 & 0.216 & 0.751 & 0.000 & 0.249 \\
\hline Amusement & Excitement & 0.5 & 0.694 & 0.244 & 0.388 & 0.000 & 0.612 \\
\hline Amusement & Socialization & 1 & 0.784 & 0.250 & 0.571 & 0.002 & 0.427 \\
\hline Excitement & Socialization & 0.5 & 0.513 & 0.136 & 0.051 & 0.024 & 0.925 \\
\hline
\end{tabular}

\section{Dominance Analysis of Cognition Facets}

The logistic dominance analysis with bootstrapping for the gambling cognition facets is shown in Table 3. In the original sample, inability to stop dominated interpretive bias, expectancies, illusion of control, and predictive control. It should be noted that each of these findings had high reproducibility as inability to stop was the most dominant facet in the majority of repeated samples as well. Interpretive bias and expectancies both dominated illusion of control and predictive control. Although dominance could not be established between interpretive bias and expectancies, expectancies dominated interpretive bias in $40.6 \%$ of the repeated samples whereas interpretive bias dominated expectancies in only 
$6.3 \%$ of the repeated samples. That dominance pattern suggests that the expectancies facet is more dominant than interpretive bias. Finally, dominance between illusion of control and predictive control could not be established in the original sample or in $87.8 \%$ of the repeated samples, indicating that these two facets are equally important in predicting harms of problem gambling.

Table 3

Logistic dominance analysis of the gambling cognition facets on gambling harm. $D_{i j}$ values in the sample and their means $\left(\bar{D}_{i j}\right)$, standard errors, probabilities of dominating one another, and reproducibility over $S=1,000$ Bootstrap Samples

\begin{tabular}{|c|c|c|c|c|c|c|c|}
\hline i & $\mathbf{j}$ & $D_{i j}$ & $\bar{D}_{i j}$ & $S E\left(D_{i j}\right)$ & $\mathbf{P}_{\mathrm{ij}}$ & $\mathbf{P}_{\mathrm{ji}}$ & $\mathbf{P}_{\text {noij }}$ \\
\hline Inability to Stop & Interpretive Bias & 1 & 0.936 & 0.192 & 0.891 & 0.018 & 0.091 \\
\hline Inability to Stop & Expectancies & 1 & 0.813 & 0.323 & 0.717 & 0.091 & 0.192 \\
\hline Inability to Stop & Illusion of Control & 1 & 0.989 & 0.073 & 0.978 & 0.000 & 0.022 \\
\hline Inability to Stop & Predictive Control & 1 & 0.977 & 0.108 & 0.954 & 0.001 & 0.045 \\
\hline Interpretive Bias & Expectancies & 0.5 & 0.329 & 0.297 & 0.063 & 0.406 & 0.531 \\
\hline Interpretive Bias & Illusion of Control & 1 & 0.714 & 0.264 & 0.444 & 0.017 & 0.539 \\
\hline Interpretive Bias & Predictive Control & 1 & 0.725 & 0.334 & 0.549 & 0.099 & 0.352 \\
\hline Expectancies & Illusion of Control & 1 & 0.803 & 0.253 & 0.614 & 0.008 & 0.378 \\
\hline Expectancies & Predictive Control & 1 & 0.765 & 0.263 & 0.544 & 0.014 & 0.442 \\
\hline Illusion of Control & Predictive Control & 0.5 & 0.470 & 0.172 & 0.031 & 0.091 & 0.878 \\
\hline
\end{tabular}

\section{Dominance Analysis Comparing Impulsivity, Motivation,} and Cognition

A final analysis was conducted to determine the relative importance of the most dominant facets of impulsivity, gambling motivation, and gambling cognitions. As indicated by the separate dominance analysis of those factors, the facets compared were negative urgency, inability to stop, and motivation through avoidance. First, we tested the fit of the subset models. Recall that $R_{m}^{2}$ is used as the measure of model fit for logistic regression.

The results are shown in Table 4 where the rows correspond to the subset models shown in the first column of the table. The second column of the table shows the $R^{2}{ }_{m}$ measure associated with each subset model, and the remaining columns show the additional contribution of each predictor to each subset model. Table 4 shows that inability to stop made the highest additional contribution to the null model $\left(R_{m}^{2}=\right.$ $0.151)$. Furthermore, comparison of inability to stop and motivation to avoid for the model subset with negative urgency demonstrated that inability to stop contributed more to this model subset, increasing $R_{m}^{2}$ by 0.127 , than motivation to avoid did, which increased $R_{m}^{2}$ by 0.067 . Additionally, inability to stop increased $R_{m}^{2}$ by 0.091 for the model subset including avoidance whereas negative urgency increased $R_{m}^{2}$ of this model subset by 0.012 , indicating that inability to stop contributed more to this model compared to negative urgency. Therefore, inability to stop dominates the other two predictors. Similarly, motivation to avoid dominates negative urgency.

\section{Table 4}

Model fit for logistic regression with experiencing harm as the dependent variable. Inability to stop (INA), Negative Urgency $(N U)$, and Avoidance (AVOID) are the predictors

\begin{tabular}{|l|l|l|l|l|}
\hline & \multicolumn{4}{|c|}{ Additional Contribution } \\
\hline Subset Model & $\boldsymbol{R}^{\boldsymbol{2}}{ }_{\boldsymbol{m}}$ & $\mathbf{I N A}$ & NU & AVOID \\
\hline Null Model & 0.000 & 0.151 & 0.039 & 0.094 \\
\hline INA & 0.151 & & 0.015 & 0.034 \\
\hline NU & 0.039 & 0.127 & & 0.067 \\
\hline AVOID & 0.094 & 0.091 & 0.012 & \\
\hline Average level 1 & & 0.109 & 0.014 & 0.051 \\
\hline
\end{tabular}

\begin{tabular}{|l|l|l|l|l|}
\hline INA + NU & 0.166 & & & 0.025 \\
\hline INA + AVOID & 0.185 & & 0.006 & \\
\hline NU + AVOID & 0.106 & 0.085 & & \\
\hline Average level 2 & & 0.085 & 0.006 & 0.025 \\
\hline INA + NU + AVOID & 0.191 & & & \\
\hline
\end{tabular}

Table 5 summarizes the dominance analysis with bootstrapping. In the original sample, the inability to stop dominated both avoidance and negative urgency. Furthermore, avoidance dominated negative urgency. These findings suggest that the inability to stop is the most dominant predictor, followed by avoidance and then positive urgency. Each of these results had high reproducibility, as the same dominance scores were found in the majority of repeated samples.

\section{Table 5}

Logistic dominance analysis of the most dominant facets for their impact on experiencing the harms of problem gambling. $D_{i j}$ values in the sample and their means $\left(\bar{D}_{i j}\right)$, standard errors, probabilities of dominating one another, and reproducibility over $S=1,000$ Bootstrap Samples

\begin{tabular}{|l|l|l|l|l|l|l|l|}
\hline $\mathbf{i}$ & $\mathbf{j}$ & $\boldsymbol{D}_{i j}$ & $\overline{\boldsymbol{D}}_{i j}$ & $\boldsymbol{S E}\left(\boldsymbol{D}_{i j}\right)$ & $\mathbf{P}_{\mathbf{i j}}$ & $\mathbf{P}_{\mathbf{j i}}$ & $\mathbf{P}_{\text {noij }}$ \\
\hline Inability to Stop & $\begin{array}{l}\text { Negative } \\
\text { Urgency }\end{array}$ & 1 & 0.996 & 0.061 & 0.994 & 0.003 & 0.003 \\
\hline Inability to Stop & Avoidance & 1 & 0.909 & 0.273 & 0.892 & 0.074 & 0.034 \\
\hline Negative Urgency & Avoidance & 0 & 0.126 & 0.274 & 0.056 & 0.805 & 0.139 \\
\hline
\end{tabular}

Lastly, we conducted a logistic regression with inability to stop, motivation to avoid, and negative urgency as the predictors to understand the relationship between each of these predictors and experiencing harm. The logistic regression analysis showed that each of the predictors had a positive association with Harm score. As shown in table 6, inability to stop and motivation to avoid were both significant predictors of experiencing harm. Overall, this model accurately predicted whether or not an individual experienced gambling harms $68.3 \%$ of the time when using a threshold of 0.5 (i.e., predicted probabilities of experiencing harm greater than 0.5 were classified as a gambler experiencing harms). 


\section{Table 6}

Logistic regression with experiencing harm (i.e., Harm score > 0) as the response and the most dominant facets as predictors.

\begin{tabular}{|l|l|l|l|l|}
\hline Predictor & $\boldsymbol{b}$ & Std. Error & $\boldsymbol{z}$ & $\boldsymbol{p}$ \\
\hline Inability to Stop & 0.918 & 0.221 & 4.155 & $3.250 \mathrm{E}-05$ \\
\hline Avoidance & 0.531 & 0.201 & 2.643 & 0.008 \\
\hline Negative Urgency & 0.346 & 0.263 & 1.316 & 0.188 \\
\hline
\end{tabular}

\section{Discussion}

Gamblers are heterogeneous in their susceptibility to the harms of gambling. Some theoretical accounts of the sources of that variability focus on individual differences in trait impulsivity, gambling related cognitions, and motivations for gambling (Devos et al., Hearn et al.,) [11-14]. Understanding how specific combinations of those factors contribute to the likelihood of experiencing harm may lead to targeted interventions that dampen that likelihood. The purpose of the present study was to introduce dominance analysis (Azen \& Budescu) as a statistical technique to identify the relative importance of impulsivity, gambling cognitions, and gambling motivations as risk factors for gambling harms [17].

The essence of dominance analysis is to compare all possible pairings of predictors in terms of variance accounted for in the response across all possible sub-models of regression analyses. As a first step, we identified the dominant facet among five facets of impulsivity, gambling cognitions, and gambling motivations. Those dominant facets were negative urgency (a tendency to act rashly when upset), a perceived inability to stop gambling, and avoidance of negative emotions or stresses. Then we compared the relative importance of those three facets and found that the perceived inability to stop gambling was the dominant predictor followed by avoidance motivation and negative urgency. Interventions designed to mitigate harm might then be structured to address primarily the specific cognition about the inability to stop by employing techniques common to cognitive-behavior therapy approaches (Tolchard) [28]. Motivations and emotion regulation would be addressed secondarily.

There are several limitations of the current study that caution against generalization. The gamblers in the current study were recruited at a casino where electronic gaming machines (EMG's) provided the only type of gambling. It is possible that the dominant facets for gamblers who prefer other forms of gambling that are judged to involve more skill (e.g., poker; sports betting) may differ from those identified in the present study. The potential facets of the predictors and their relative dominance may vary across different measures of impulsivity (e.g., Callan et al., Patton et al.,) gambling cognitions (e.g., McInnes et al., Steenbergh et al.,) and gambling motivation (e.g., Shinaprayoon et al., Stewart \& Zack) [29-33]. We note that whereas the cognitions and motivations measured in this study were contextualized to gambling, impulsivity was measured as a context-free trait. Measures of impulsivity more directly attuned to gambling may increase its dominance as a risk factor for gambling harm.

Finally, there have been significant advances in the quantification of gambling harm. For example, Shannon et al [34]. measured seven domains of gambling harm (health; leisure; critical events; social; employment; financial; psychological) with multiple indicators for each domain. The scores on that measure correlated .65 with overall PGSI scores, but there was no direct comparison with the five adverse consequences items used in the present study. It should be noted that Ferris and Wynne based their selection of single items for personal and social adverse consequences on psychometric analysis of several items for each of their domains [4]. It is not clear whether redundancies exist in the more extensive scales tested by Shannon et al. [34]. Future developments may establish a new gold standard for measuring gambling harms. Theoretically, it remains important to identify the critical facets of risk factors such as impulsivity, cognitions, and motivation as those factors may be central to other forms of addiction (Dong \& Potenza). More practically, assessing the relative dominance of those factors may guide the structure of interventions designed to reduce experiencing harms from gambling [35].

\section{References}

1. Calado F, Griffiths M (2016) Problem gambling worldwide: An update and systematic review of empirical research (20002015). Journal of Behavioral Addictions 5: 592-613.

2. Canale N, Vieno A, Griffiths M (2016) The extent and distribution of gambling-related harms and the prevention paradox in a British population survey. (FULL-LENGTH REPORT) (Report) 5: 204-212.

3. Delfabbro P, King D, Browne M, Dowling N (2020) Do EGMs have a stronger association with problem gambling than racing and casino table games? Evidence from a decade of Australian prevalence studies. Journal of Gambling Studies 36:499-511.

4. Ferris JA, Wynne HJ (2001) The Canadian problem gambling index. Canadian Centre on Substance Abuse Ottawa, ON.

5. Nelson S, LaPlante D, Gray H, Tom M, Kleschinsky J, Shaffer $\mathrm{H}$, et al. (2018) Already at the table: Patterns of play and gambling involvement prior to gambling expansion. Journal of Gambling Studies 34: 275-295.

6. Chiu J, Storm L (2010) Personality, Perceived luck and gambling attitudes as predictors of gambling involvement. Journal of Gambling Studies 26: 205-227.

7. Maclaren V, Fugelsang J, Harrigan K, Dixon M (2011) The personality of pathological gamblers: A meta-analysis. Clinical Psychology Review 31: 1057-1067.

8. Michalczuk R, Bowden-Jones H, Verdejo-Garcia A, Clark L (2011) Impulsivity and cognitive distortions in pathological gamblers attending the UK National Problem Gambling Clinic: a preliminary report. Psychological Medicine 41: 2625-2635.

9. Odlaug BL, Schreiber LRN, Grant JE (2013) Personality dimensions and disorders in pathological gambling, Current Opinion in Psychiatry 6: 107-112.

10. Passanisi A, Pace U (2017) The unique and common contributions of impulsivity and decision-making strategies 
among young adult Italian regular gamblers. Personality and Individual Differences 105: 24-29.

11. Devos M, Clark L, Bowden-Jones H, Grall-Bronnec M, Challet-Bouju G, et al. (2020). The joint role of impulsivity and distorted cognitions in recreational and problem gambling: A cluster analytic approach. Journal of Affective Disorders 260: 473-482.

12. Goodie A, Fortune E (2013) Measuring cognitive distortions in pathological gambling: Review and meta-analyses. Psychology of Addictive Behaviors 27: 730-743.

13. Myrseth H, Brunborg G, Eidem M (2010) Differences in cognitive distortions between pathological and nonpathological gamblers with preferences for chance or skill games. Journal of Gambling Studies 26: 561-569.

14. Hearn N, Ireland J, Eslea M, Fisk J (2020) Exploring pathways to gambling: Proposing the integrated risk and protective factors model of gambling types. Journal of Gambling Studies. https://doi.org/10.1007/s10899-020-09929-2.

15. Caler K, Garcia J, Nower L (2016) Assessing problem gambling: A review of classic and specialized measures. Current Addiction Reports 3: 437-444.

16. Miller N, Currie S, Hodgins D, Casey D (2013) Validation of the problem gambling severity index using confirmatory factor analysis and rasch modelling. International Journal of Methods in Psychiatric Research 22: 245-255.

17. Azen R, Budescu D (2003) The dominance analysis Approach for comparing predictors in multiple regression. Psychological Methods 8: 129-148.

18. Wynne H (2003) Introducing the Canadian Problem Gambling Index. Edmonton, AB: Wynne Resources.

19. Currie S, Hodgins D, Casey D (2013) Validity of the Problem Gambling Severity Index interpretive categories. Journal of Gambling Studies 29: 311-327.

20. Cyders M, Smith G, Spillane N, Fischer S, Annus A, et al. (2007) Integration of impulsivity and positive mood to predict risky behavior: Development and validation of a measure of positive urgency. Psychological Assessment 19: 107-118.

21. Lee H, Chae P, Lee H, Kim Y (2007) The five-factor gambling motivation model. Psychiatry Research 150: 21-32.

22. Raylu N, Oei T (2004) The Gambling Related Cognitions Scale (GRCS): development, confirmatory factor validation and psychometric properties. Addiction 99: 757-769.
23. Delaney HD: Partial correlation. In: Salkind NJ (ed.) Encyclopedia of Research Design, pp. 1003-1006. SAGE Publications, Thousand Oaks (2010)

24. Vargha A, Bergman L, Delaney H (2013) Interpretation problems of the partial correlation with nonnormally distributed variables. Quality \& Quantity 47: 3391-3402.

25. Mcfadden D (1974) The measurement of urban travel demand. Journal of Public Economics 3:303-328.

26. Azen R, Traxel N (2009) Using dominance analysis to determine predictor importance in logistic regression. Journal of Educational and Behavioral Statistics 34: 319-347.

27. Menard S (2000) Coefficients of determination for multiple logistic regression analysis. The American Statistician 54: 17-24.

28. Tolchard B (2017) Cognitive-behavior therapy for problem gambling: a critique of current treatments and proposed new unified approach. Journal of Mental Health 26: 283-290.

29. Patton JH, Stanford MS, Barratt ES (1995) Factor structure of the Barratt Impulsiveness Scale. Journal of Clinical Psychology 51: 768-774.

30. McInnes A, Hodgins DC, Holub A (2014) The Gambling Cognitions Inventory: scale development and psychometric validation with problem and pathological gamblers. International Gambling Studies 14: 410-431.

31. Steenbergh T, Meyers A, May R, Whelan J (2002) Development and Validation of the Gamblers' Beliefs Questionnaire. Psychology of Addictive Behaviors 16: 143-149.

32. Shinaprayoon T, Carter NT, Goodie AS (2017) The modified gambling motivation scale: Confirmatory factor analysis and links with problem gambling. Journal of Gambling 37: 108-135.

33. Stewart S, Zack M (2008) Development and psychometric evaluation of a three-dimensional Gambling Motives Questionnaire. Addiction 103: 1110-1117.

34. Shannon K, Anjoul F, Blaszczynski A (2017) Mapping the proportional distribution of gambling-related harms in a clinical and community sample. International Gambling Studies 17: 366-385.

35. Dong G, Potenza MN (2014) A cognitive-behavioral model of Internet gaming disorder: Theoretical underpinnings and clinical implications. Journal of Psychiatric Research 58: 7-11.

Copyright: (C2020 Harvey HC Marmurek. This is an open-access article distributed under the terms of the Creative Commons Attribution License, which permits unrestricted use, distribution, and reproduction in any medium, provided the original author and source are credited. 